\title{
A General Setting for the Pointwise Investigation of Determinacy
}

\author{
Yurii Khomskii ${ }^{\star}$ \\ Institute of Logic, Language and Computation \\ University of Amsterdam \\ Plantage Muidergracht 24 \\ 1018 TV Amsterdam \\ The Netherlands
}

\begin{abstract}
It is well-known that if we assume a large class of sets of reals to be determined then we may conclude that all sets in this class have certain regularity properties: we say that determinacy implies regularity properties classwise. In [Lö05] the pointwise relation between determinacy and certain regularity properties (namely the Marczewski-Burstin algebra of arboreal forcing notions and a corresponding weak version) was examined.

An open question was how this result extends to topological forcing notions whose natural measurability algebra is the class of sets having the Baire property. We study the relationship between the two cases, and using a definition which adequately generalizes both the MarczewskiBurstin algebra of measurability and the Baire property, prove results similar to [Lö05].

We also show how this can be further generalized for the purpose of comparing algebras of measurability of various forcing notions.
\end{abstract}

\section{Introduction}

The classical theorems due to Mycielski-Swierczkowski, Banach-Mazur and Morton Davis respectively state that under the Axiom of Determinacy all sets of reals are Lebesgue measurable, have the Baire property and the perfect set property (see, e.g., [Ka94, pp 373-377]). In fact, these proofs give classwise implications, i.e., if $\boldsymbol{\Gamma}$ is a boldface pointclass (closed under continuous preimages and intersections with basic open sets) such that all sets in $\boldsymbol{\Gamma}$ are determined, then all sets in $\boldsymbol{\Gamma}$ have the corresponding regularity property. The proofs do not, however, show that from the assumption " $A$ is determined" one can conclude " $A$ is regular", i.e., they do not give us pointwise implications. So a natural question is: what is the strength of the statement " $A$ is determined", and which properties of $A$ follow from that statement?

\footnotetext{
* The author is supported from a Mosaic grant (no 017.004.066) by the Netherlands
} Organisation for Scientific Research (NWO). 
That the strength of determinacy is in classwise rather than pointwise consequences is not unexpected - after all, it is easy to construct sets that are determined for trivial reasons. Still, if the regularity properties themselves are "weak" in a certain sense, the relationship is not as clear. In [Lö05], where the pointwise relationship between determinacy and Marczewski-Burstin measurability algebras (connected to so-called arboreal forcing notions) was first analyzed, we indeed have the expected result for the algebras themselves but more interesting ones if we consider their "weak" or "local" counterparts.

This investigation started with the question whether the results of [Lö05] can be transferred to the more difficult scenario of topological forcing notions whose natural algebra of measurability is not the Marczewski-Burstin algebra but the Baire property in the corresponding topology. In the process of studying this question, however, certain basic properties of arboreal forcings and their measurability algebras came to light, which forced us to adapt the definitions as well as the actual question. Partly motivated by recent work of Daisuke Ikegami [Ik08], we are adapting a different definition of arboreal forcings, and giving a new definition of a measurability notion. Using these new definitions we are able to generalize and improve [Lö05] while covering both the non-topological and the new topological cases. Our two main results here are Theorem 4.3 and Theorem 5.5.

In the last section we also show how the methods can be generalized for the purpose of comparing algebras of measurability of various forcing notions.

We should note that Definition 2.2 below gives far less freedom than [Lö05, Section 2.1], but there are good reasons for adopting it: firstly, Fact 2.3 could not be proved without it, secondly, one would be able to construct some very simple sets (e.g., closed in the standard topology) that are non-measurable. In short, the new definition eliminates "pathological cases" and makes sure that our forcing notions are somewhat reasonable. This, of course, also eliminates most of the crucial examples considered in [Lö05, Sections 5 and 6]. As a result, our conclusions differ from [Lö05] on certain points, but we feel that the new analysis is more intuitively satisfying and has more practical relevance because it is immune to artificial counterexamples.

\section{Definitions and Preliminaries}

We start by fixing some simple concepts about descriptive-theoretic trees:

Definition 2.1. Let $T \subseteq \omega^{<\omega}$ or $2^{<\omega}$ be a tree.

1. For $t \in T$ we write $\operatorname{Succ}_{T}(t):=\{s \in T \mid \exists n(s=t \frown\langle n\rangle)\}$ to denote the set of immediate successors of $t$.

2. A node $t \in T$ is called

- splitting if $\left|\operatorname{Succ}_{T}(t)\right|>1$ and non-splitting otherwise.

$-\omega$-splitting if $\left|\operatorname{Succ}_{T}(t)\right|=\omega$ and $n$-splitting if $\left|\operatorname{Succ}_{T}(t)\right|=n<\omega$. 
- totally splitting if $\forall n(t \frown\langle n\rangle \in T)$.

3. The stem of $T$, notation $\operatorname{stem}(T)$, is the largest $s \in T$ such that all $t \subseteq s$ are non-splitting.

4. $[T]$ denotes the set of branches through T, i.e., $\{x \mid \forall n(x \mid n \in T)\}$.

Although we try to keep the notions $T$ and $[T]$ separated, occasionally we will use the two objects interchangeably, since it makes arguments simpler and can cause no harm. Finally, we note that all trees considered in this paper are assumed to be pruned, i.e., every node has at least one successor.

We are ready to define arboreal forcing notions:

Definition 2.2. A forcing partial order $(\mathbb{P}, \leq)$ is called arboreal if it is a collection of perfect trees on $\omega^{\omega}$ (or $\left.2^{\omega}\right)$, ordered by inclusion, with the extra condition that

$$
\forall P \in \mathbb{P} \forall t \in P \exists Q \leq P(t \subseteq \operatorname{stem}(Q)) .
$$

It is called topological if the set of conditions $\{[P] \mid P \in \mathbb{P}\}$ forms a topology base for some topology on the set $\omega^{\omega}$ (resp. $\left.2^{\omega}\right)$, and non-topological otherwise.

Examples of standard non-topological arboreal forcings include Sacks forcing, Miller forcing, Laver forcing, Silver forcing and many more (for a definition see e.g. [BaJu95,Je86].) Examples of standard topological forcings are Cohen forcing, Hechler forcing, eventually different forcing and Matthias forcing. Cohen forcing generates the standard topology, while Hechler and eventually different forcing generate the dominating topology and the eventually different topology, respectively. Matthias forcing generates the Ellentuck topology (due to Erik Ellentuck [El74]).

The following fact is a straightforward consequence of our definition.

Lemma 2.3. If $\mathbb{P}$ is an arboreal forcing notion, then $\mathbb{P}$ is separative. Moreover, we have for all $P, Q \in \mathbb{P}$, if $P \not \leq Q$ then $\exists R \leq P$ s.t. $[R] \cap[Q]=\varnothing$ (we say $\mathbb{P}$ is strongly separative.)

Proof. Suppose $P \not \leq Q$. Then there is $t \in P \backslash Q$, so by definition there must be an $R \leq P$ with $t \subseteq \operatorname{stem}(R)$. But then $[R] \cap[Q]=\varnothing$.

Since this paper is about consequences of determinacy, let us also give that definition. There are a number of equivalent formulations of determinacy but for our purposes the most convenient is to use the following:

\section{Definition 2.4.}

1. A tree $\sigma$ is called a strategy for player I if all nodes of odd length are totally splitting and all nodes of even length are non-splitting.

2. A tree $\tau$ is called a strategy for player II if all nodes of even length are totally splitting and all nodes of odd length are non-splitting. 
3. A set $A \subseteq \omega^{\omega}$ is called determined if there is either a strategy $\sigma$ for player $I$ such that $[\sigma] \subseteq A$ or a strategy $\tau$ for player II such that $[\tau] \subseteq A^{\mathrm{c}}$.

Since by [So70] it is consistent with ZF that all sets of reals have the regularity properties, the only way to prove a non-trivial pointwise connection between determinacy and these properties is by using AC. The way one would typically prove that there are sets that are, e.g., non-Lebesgue measurable, don't have the Baire property, the perfect set property etc. is by a diagonalization procedure called the Bernstein construction. In the most general setting this is the following fact:

Theorem 2.5. (General Bernstein Theorem) Let $\left\{X_{\alpha} \mid \alpha<2^{\aleph_{0}}\right\}$ be a collection of $2^{\aleph_{0}}$ sets of reals, such that $\left|X_{\alpha}\right|=2^{\aleph_{0}}$ for all $\alpha$. Then there are disjoint sets $A, B \subseteq \bigcup_{\alpha<2^{\aleph_{0}}} X_{\alpha}$, called the Bernstein components, such that for all $\alpha<2^{\aleph_{0}}$, $X_{\alpha} \cap A \neq \varnothing$ and $X_{\alpha} \cap B \neq \varnothing$.

\section{Marczewski-Burstin algebras, the Baire property and Measurability}

It is natural to connect each arboreal forcing notion $\mathbb{P}$ to a corresponding regularity property, or a so-called algebra of measurability. For example, random forcing (considered as the collection of perfect trees with non-null Lebesgue measure) is naturally connected to Lebesgue-measurability, and Cohen forcing to the Baire property in the standard topology on $\omega^{\omega}$. In analogy with the latter case, Hechler and eventually different forcing are connected to the Baire properties in the dominating and eventually different topologies on $\omega^{\omega}$, respectively.

For the non-topological arboreal forcings, the regularity property usually considered has been the Marczewski-Burstin algebra.

Definition 3.1. Let $\mathbb{P}$ be arboreal and $A \subseteq \omega^{\omega}$.

1. $A$ is called $\mathbb{P}$-Marczewski-Burstin-measurable if $\forall P \in \mathbb{P} \exists Q \leq P([Q] \subseteq$ $\left.A \vee[Q] \subseteq A^{\mathrm{c}}\right)$.

2. $A$ is called $\mathbb{P}$-null if $\forall P \in \mathbb{P} \exists Q \leq P[Q] \subseteq A^{\mathrm{c}}$.

3. $A$ is called $\mathbb{P}$-meager if it is a countable union of $\mathbb{P}$-null sets.

We denote the class of $\mathbb{P}$-Marczewski-Burstin-measurable sets by $\mathrm{MB}(\mathbb{P})$, the ideal of $\mathbb{P}$-null sets by $\mathcal{N}_{\mathbb{P}}$ and the $\sigma$-ideal of $\mathbb{P}$-meager sets by $\mathcal{I}_{\mathbb{P}}$. Note that when $\mathbb{P}$ is topological then $\mathbb{P}$-null is the same as being nowhere dense in the $\mathbb{P}$-topology and $\mathbb{P}$-meager is exactly the topological concept of being meager (or of first category).

For the standard non-topological forcings $\mathbb{P}$, a fusion argument like in [Je86, p $15 \mathrm{ff}]$ shows that $\mathrm{MB}(\mathbb{P})$ is a $\sigma$-algebra. The same holds for Matthias forcing, although the proof is technically more involved (see [El74]). However, for Cohen, 
Hechler or eventually different forcing, this is not the case: if we let $\mathbb{P}$ be any one of these three forcings, then, for instance, $A:=\left\{x \mid \forall^{\infty} n(x(n)\right.$ is even) $\}$ is not in $\operatorname{MB}(\mathbb{P})$. To see this, note that for all $P \in \mathbb{P}$ there exists an $x \in[P]$ which is eventually even and a $y$ which is not eventually even, so $[P] \nsubseteq A$ and $[P] \nsubseteq A^{\mathrm{c}}$. On the other hand, we can write $A=\bigcup_{N} A_{N}$ where $A_{N}:=\{x \mid \forall n \geq N(x(n)$ is even) $\}$, which is easily seen to be $\mathbb{P}$-null. So then $\operatorname{MB}(\mathbb{P})$ is not a $\sigma$-algebra, it doesn't contain $F_{\sigma}$ sets, and is in general not a regularity property at all.

It is then not at all surprising that in the topological cases, rather than $\mathrm{MB}(\mathbb{P})$ one usually considers the algebra consisting of those sets having the Baire property in the $\mathbb{P}$-topology, which we shall denote by $\mathrm{BP}(\mathbb{P})$. The definition below should shed some light on the precise reason for this dichotomy and the relationship between $\operatorname{MB}(\mathbb{P})$ and $\operatorname{BP}(\mathbb{P})$. It is close to that of the Marczewski-Burstin algebra but is more natural and well-behaved. For example, Ikegami in [Ik08] uses it to prove general theorems about the strength of projective measurability statements. We shall refer to this property simply by $\mathbb{P}$-measurability.

Definition 3.2. Let $\mathbb{P}$ be a topological arboreal forcing. For sets $A, B$ we write $A \subseteq^{*} B$ if $A \backslash B \in \mathcal{I}_{\mathbb{P}}$. Then a set $A$ is called $\mathbb{P}$-measurable if

$$
\forall P \in \mathbb{P} \exists Q \leq P\left([Q] \subseteq^{*} A \text { or }[Q] \subseteq^{*} A^{\mathrm{c}}\right) .
$$

We shall denote the class of $\mathbb{P}$-measurable sets by $\operatorname{Meas}(\mathbb{P})$.

The following are simple but important properties:

Lemma 3.3. Let $\mathbb{P}$ be arboreal.

1. For all $P \in \mathbb{P},[P]$ is not $\mathbb{P}$-meager,

2. $\operatorname{MB}(\mathbb{P}) \subseteq \operatorname{Meas}(\mathbb{P})$, and

3. $\operatorname{Meas}(\mathbb{P})=\operatorname{MB}(\mathbb{P})$ iff $\mathcal{N}_{\mathbb{P}}=\mathcal{I}_{\mathbb{P}}$.

Proof.

1. Suppose towards contradiction that $[P]=\bigcup_{n} M_{n}$ with $M_{n} \in \mathcal{N}_{\mathbb{P}}$. By induction, let $P_{0} \leq P$ s.t. $\left[P_{0}\right] \cap M_{0}=\varnothing$. Using the definition of arboreal forcings, let $P_{0}^{\prime} \leq P_{0}$ be anything with a strictly longer stem. Then let $P_{1} \leq P_{0}^{\prime}$ be s.t. $\left[P_{1}\right] \cap M_{1}=\varnothing$, etc. Then we get a sequence

$$
P \geq P_{0} \geq P_{1} \geq P_{2} \geq \ldots
$$

of trees with strictly increasing stems, hence there is a real $x:=\bigcup_{n} \operatorname{stem}\left(P_{n}\right)$. Moreover, by the general property of trees it is easy to see that $\bigcap_{n}\left[P_{n}\right]=\{x\}$. So $x \in[P]$ but $x \notin \bigcup_{n} M_{n}$ : contradiction.

2. Obvious.

3. Suppose $\mathcal{I}_{\mathbb{P}}=\mathcal{N}_{\mathbb{P}}$. If $A \in \operatorname{Meas}(\mathbb{P})$ then for $P \in \mathbb{P}$ there is $Q \leq P$ s.t. $[Q] \cap A \in \mathcal{I}_{\mathbb{P}}=\mathcal{N}_{\mathbb{P}}$ or $[Q] \backslash A \in \mathcal{I}_{\mathbb{P}}=\mathcal{N}_{\mathbb{P}}$. So then there is $R \leq Q$ s.t. $[R] \cap([Q] \cap A))=\varnothing$ resp. $[R] \subseteq([Q] \cap A))$.

Conversely, let $A \in \mathcal{I}_{\mathbb{P}}$. Since this means that $A \in \operatorname{Meas}(\mathbb{P})=\operatorname{MB}(\mathbb{P})$, for all $P$ there is $Q \leq P$ such that $[Q] \subseteq A$ or $[Q] \cap A=\varnothing$. But the former is impossible by (1). 
Here, (1) is an analogue of the classical Baire Category Theorem. Results of [Ik08] show that Meas $(\mathbb{P})$ is always a $\sigma$-algebra, and moreover that $\operatorname{Meas}(\mathbb{P})=\operatorname{BP}(\mathbb{P})$ for topological $\mathbb{P}$. Hence, the difference between the original two properties$\operatorname{MB}(\mathbb{P})$ and $\operatorname{BP}(\mathbb{P})$ - is exactly the difference between "meager" and "nowhere dense". Since from a topological point of view these concepts usually do not coincide, this explains why $\mathrm{MB}(\mathbb{P})$ usually fails to be a good regularity property for topological forcings. (Incidentally, the Ellentuck topology is a well-known example of a topology where "meager" and "nowhere dense" do coincide. Hence, if $\mathbb{P}$ is Matthias forcing then $\operatorname{BP}(\mathbb{P})=\operatorname{MB}(\mathbb{P})$, and the latter is precisely the collection of completely Ramsey sets, cf. [El74]).

In [Lö05] the Marczewski-Burstin algebra for non-topological forcing notions (and later it's weak variant) played the crucial role. We shall do the same thing for $\operatorname{Meas}(\mathbb{P})$. Since the new property is either the same or larger than those previously considered, any statement of the kind "there is a determined set which is not $\mathbb{P}$-measurable" immediately implies the same statement with "P्Pmeasurable" replaced by "P-Marcewski-Burstin-measurable" or by "having the Baire property in the $\mathbb{P}$-topology". Thus, our results are a natural generalization of [Lö05].

\section{Determinacy and Measurability}

Are there determined sets which are not in Meas $(\mathbb{P})$ ? We get the expected answer: yes. The main ingredient is, as in [Lö05], Bernstein's theorem, but we need a technical argument before we can apply it.

Lemma 4.1. Let $\mathbb{P}$ be an arboreal forcing notion. If $P \in \mathbb{P}$ and $C \subseteq[P]$ is $\mathbb{P}$-comeager in $[P]$, then there exists a perfect tree $T$ with $[T] \subseteq C$.

Proof. Let $[P] \backslash C:=\bigcup_{n} M_{n}$ with each $M_{n} \in \mathcal{N}_{\mathbb{P}}$. Let $C_{n}:=[P] \backslash M_{n}$, so that $C=\bigcap_{n} C_{n}$. By induction we shall construct a collection of $P_{u} \in \mathbb{P}$ indexed by $u \in 2^{<\omega}$, while taking care that $\left[P_{u}\right] \subseteq C_{|u|}$ for all $u$.

- Since $M_{0} \in \mathcal{N}_{\mathbb{P}}$, pick $P_{\varnothing} \leq P$ such that $P_{\varnothing} \cap M_{0}=\varnothing$, i.e., $\left[P_{\varnothing}\right] \subseteq C_{0}$.

- Suppose we have $u \in 2^{<\omega}$ with $|u|=n$, and $\left[P_{u}\right] \subseteq C_{n}$. Since $P_{u}$ is perfect we can extend its stem $t$ to two incompatible stems $t^{\prime}$ and $t^{\prime \prime}$. Since $\mathbb{P}$ is arboreal, there are $P_{u}^{\prime}$ and $P_{u}^{\prime \prime}$ such that $t^{\prime} \subseteq \operatorname{stem}\left(P_{u}^{\prime}\right)$ and $t^{\prime \prime} \subseteq \operatorname{stem}\left(P_{u}^{\prime \prime}\right)$. Now, since $M_{n+1} \in \mathcal{N}_{\mathbb{P}}$, there are $\left[P_{u \frown\langle 0\rangle}\right] \subseteq\left[P_{u}^{\prime}\right] \backslash M_{n+1}$ and $\left[P_{u-\langle 1\rangle}\right] \subseteq\left[P_{u}^{\prime \prime}\right] \backslash M_{n+1}$.

Let $T$ be the tree generated by $\left\{\operatorname{stem}\left(P_{u}\right) \mid u \in 2^{<\omega}\right\}$. By our construction, this is clearly a perfect tree, so it just remains to prove that $[T] \subseteq C$. But, for every $x \in[T]$ there is a $y \in 2^{\omega}$ such that $x=\bigcup_{n} \operatorname{stem}\left(P_{y\lceil n}\right)$. Moreover, it is easy to see that $\bigcap_{n}\left[P_{y\lceil n}\right]=\{x\}$. Therefore, for all $n$ we have $x \in\left[P_{y\lceil n}\right] \subseteq C_{n}$, hence $x \in C$. 
Corollary 4.2. Let $\mathbb{P}$ be arboreal and $A \subseteq \omega^{\omega} \mathbb{P}$-measurable. Then

$$
\forall P \in \mathbb{P} \exists T \subseteq P\left(T \text { is a perfect tree and }[T] \subseteq A \text { or }[T] \subseteq A^{\mathrm{c}}\right) .
$$

Proof. Let $A \in \operatorname{Meas}(\mathbb{P})$ and $P \in \mathbb{P}$. We know that there is a $P^{\prime} \in \mathbb{P}$ with $P^{\prime} \leq P$ such that $\left[P^{\prime}\right] \backslash A$ is meager or $\left[P^{\prime}\right] \cap A$ is meager. In the former case $C:=A \cap\left[P^{\prime}\right]$ is comeager so there is a perfect tree in $A$, and in the latter case $\left[P^{\prime}\right] \backslash A$ is comeager so there is a perfect tree in $A^{\mathrm{c}}$.

The corollary is sufficient to construct a counterexample using a Bernstein diagonalization procedure:

Theorem 4.3. Determinacy does not imply $\mathbb{P}$-measurability pointwise.

Proof. Fix any $P \in \mathbb{P}$ with $|\operatorname{stem}(P)| \geq 2$. Then fix any strategy $\sigma$ such that $[P] \cap[\sigma]=\varnothing$, which is always possible just by letting the beginning of $\sigma$ be different from the stem of $P$. Then let $\left\langle T_{\alpha} \mid \alpha<2^{\aleph_{0}}\right\rangle$ be an enumeration of all perfect trees in $[P]$. By the general Bernstein theorem 2.5 there are disjoint sets $A, B \subseteq \bigcup_{\alpha<2^{\aleph_{0}}}\left[T_{\alpha}\right] \subseteq[P]$, both of which intersect every $T_{\alpha}$. Let $A^{\prime}:=A \cup[\sigma]$. Then, by the contraposition of Corollary 4.2 , neither $A^{\prime}$ nor $A^{\prime c}$ is in $\operatorname{Meas}(\mathbb{P})$, but clearly either $A^{\prime}$ or $A^{\prime c}$ is determined (the former if $\sigma$ was a strategy for player $I$ and the latter if it was for player $I I)$.

\section{Determinacy and weak Measurability}

In [Lö05], the question became more interesting when instead of full measurability one considered a weak, or local version.

Definition 5.1. Let $\mathbb{P}$ be arboreal, and let $A \subseteq \omega^{\omega}$. Then

1. $A$ is weakly $\mathbb{P}$-Marczewski-Burstin-measurable if $\exists P \in \mathbb{P}$ s.t. $[P] \subseteq A$ or $[P] \subseteq A^{\mathrm{c}}$

2. $A$ is weakly $\mathbb{P}$-measurable if $\exists P \in \mathbb{P}$ s.t. $[P] \subseteq^{*} A$ or $[P] \subseteq^{*} A^{\mathrm{c}}$.

We denote the class of weakly $\mathbb{P}$-Marczewski-Burstin-measurable sets by ${ }_{w M B}(\mathbb{P})$ and the class of weakly $\mathbb{P}$-measurable sets by wMeas $(\mathbb{P})$. An important reason for introducing this property is that it is classwise equivalent to full measurability. By [BrLö99, Lemma 2.1] $\mathrm{MB}(\mathbb{P})$ and $\mathrm{wMB}(\mathbb{P})$ are classwise equivalent for all standard $\mathbb{P}$ and all topologically reasonable pointclasses. We will prove the same for $\operatorname{Meas}(\mathbb{P})$ and wMeas $(\mathbb{P})$, plus, we will make precise which condition on $\mathbb{P}$ is required for this equivalence to hold.

Definition 5.2. Let $\mathbb{P}$ be an arboreal forcing. We say that $\mathbb{P}$ is topologically homogeneous if for every $P \in \mathbb{P}$ there is a homeomorphism $f_{P}: \omega^{\omega} \stackrel{\sim}{\longrightarrow}[P]$, in the sense of the standard topology, such that for every tree $T$ we have $T \in \mathbb{P}$ iff the tree of $f_{P}[T]$ is in $\mathbb{P}$. 
It can be shown that all the standard examples of arboreal forcing notions $\mathbb{P}$ are topologically homogeneous.

Lemma 5.3. Let $\mathbb{P}$ be topologically homogeneous and $P \in \mathbb{P}$. Then $A$ is $\mathbb{P}$ meager iff $f_{P}[A]$ is $\mathbb{P}$-meager.

Proof. Since $f_{P}$ is a bijection, it is sufficient to prove the claim for $\mathbb{P}$-meager replaced by $\mathbb{P}$-null. We show that if $A$ is $\mathbb{P}$-null then $f_{P}[A]$ is $\mathbb{P}$-null-for the converse direction, use $f_{P}^{-1}$. Let $Q \in \mathbb{P}$ be arbitrary. We must show that there is an $R \leq Q$ s.t. $[R] \cap f_{P}[A]=\varnothing$. Since $\mathbb{P}$ is strongly separative, we may assume w.l.o.g. that $Q \leq P$. Then the tree of $f_{P}^{-1}[Q]$ is a member of $\mathbb{P}$, so by assumption there exists an $R^{\prime} \leq f_{P}^{-1}[Q]$ s.t. $\left[R^{\prime}\right] \cap A=\varnothing$. Then let $R:=$ the tree of $f_{P}\left[R^{\prime}\right]$, so $R \leq Q$ and $[R] \cap f_{P}[A]=\varnothing$.

Theorem 5.4. Let $\mathbb{P}$ be a topologically homogeneous arboreal forcing notion and let $\boldsymbol{\Gamma}$ be a pointclass closed under continuous preimages and intersections with closed sets. Then $\boldsymbol{\Gamma} \subseteq \operatorname{Meas}(\mathbb{P})$ iff $\boldsymbol{\Gamma} \subseteq$ wMeas $(\mathbb{P})$.

Proof. The forward direction is obvious. For the backward direction, let $A \in \boldsymbol{\Gamma}$. Fix a $P \in \mathbb{P}$, and we must show that there is a $Q \leq P$ such that $[Q] \subseteq^{*} A$ or $[Q] \subseteq^{*} A^{\mathrm{c}}$. By the assumption on $\boldsymbol{\Gamma}$, we know that $A \cap[P] \in \boldsymbol{\Gamma}$ and hence $A^{\prime}:=f_{P}^{-1}([A] \cap P) \in \Gamma$. By assumption, there exists a $Q^{\prime} \in \mathbb{P}$ s.t. $\left[Q^{\prime}\right] \subseteq^{*} A^{\prime}$ or $\left[Q^{\prime}\right] \subseteq^{*} A^{\prime c}$. Let $Q$ be the tree of $f_{P}\left[Q^{\prime}\right]$. Then $Q \leq P$ and by Lemma 5.3 $[Q] \subseteq^{*} A$ or $[Q] \subseteq^{*} A^{\mathrm{c}}$.

In [Lö05], the arboreal forcings $\mathbb{P}$ were classified into three groups, in such a way that in the first case determinacy implied $w \mathrm{mB}(\mathbb{P})$ pointwise, in the second case it did not, and in the third there were examples either way. As we noted in the introduction, we are adopting a stricter definition of arboreal forcing notions which eliminates the pathological examples from [Lö05]. As a result, we are now able to give an exhaustive characterization.

First, we fix an arboreal forcing $\mathbb{P}$. Then we split the situation into two cases:

- Case 1: For every strategy $\sigma$ there exists $P \in \mathbb{P}$ s.t. $P \subseteq \sigma$.

- Case 2: For some strategy $\sigma$, the set $[\sigma]$ is $\mathbb{P}$-null.

Let us immediately check why this case distinction is exhaustive: suppose Case 1 doesn't hold, so there exists a $\sigma$ s.t. there is no $P \subseteq \sigma$. But then, for every $P \in \mathbb{P}$ there is a $t \in P \backslash \sigma$ and consequently $Q \leq P$ with $t \subseteq \operatorname{stem}(Q)$. So $[Q] \cap[\sigma]=\varnothing$ and we are in Case 2. Conversely, if $[\sigma]$ is $\mathbb{P}$-null then $\sigma$ clearly cannot contain any $P \in \mathbb{P}$.

Theorem 5.5. In Case 1, Determinacy implies wMeas $(\mathbb{P})$ pointwise. In Case 2, Determinacy does not imply wMeas $(\mathbb{P})$ pointwise. 
Proof.

Case 1. Suppose $A$ is determined. Then there is a strategy $\sigma$ s.t. $[\sigma] \subseteq A$ or $[\sigma] \subseteq A^{\mathrm{c}}$. It follows immediately that there is a $P \in \mathbb{P}$ s.t. $[P] \subseteq A$ or $[P] \subseteq A^{\mathrm{c}}$, so $A$ is certainly in wMeas $(\mathbb{P})$.

Case 2. Fix a strategy $\sigma$ which is $\mathbb{P}$-null. Let $\mathfrak{T}_{\neg \sigma}$ be the collection of perfect trees disjoint from $[\sigma]$.

Claim. For every $A \in \mathrm{wMeas}_{(\mathbb{P})}$ there is $T \in \mathfrak{T}_{\neg \sigma}$ such that $[T] \subseteq A$ or $[T] \subseteq A^{\mathrm{c}}$.

Proof. First, suppose there is a $P \in \mathbb{P}$ such that $[P] \subseteq^{*} A^{\text {c }}$, i.e., $[P] \cap A \in \mathcal{I}_{\mathbb{P}}$. Since $[\sigma]$ is $\mathbb{P}$-null, there is a $Q \leq P$ such that $[Q] \cap[\sigma]=\varnothing$. Then $C:=[Q] \backslash A$ is $\mathbb{P}$-comeager in $[Q]$ and disjoint from $[\sigma]$. So by Lemma 4.1 there is a perfect tree $[T] \subseteq C$. Then $[T] \subseteq A^{\mathrm{c}}$ and $T$ is disjoint from $[\sigma]$, so $T \in \mathfrak{T}_{\neg \sigma}$. Now, the case where $[P] \subseteq^{*} A$ is analogous.

$\square$ (Claim)

Since $\mathfrak{T}_{\neg \sigma}$ is a collection of $2^{\aleph_{0}}$ sets of size $2^{\aleph_{0}}$, we can use the general Bernstein theorem 2.5 to find disjoint sets $A$ and $B$ intersecting every member of $\mathfrak{T}_{\neg \sigma}$. Note that by construction, both $A$ and $B$ are disjoint from $[\sigma]$. Now let $A^{\prime}:=A \cup[\sigma]$. Then, by the contraposition of the Claim, neither $A^{\prime}$ nor $A^{\prime c}$ is in wMeas $(\mathbb{P})$ but clearly either $A^{\prime}$ or $A^{\prime c}$ is determined (again depending on whether $\sigma$ was a strategy of player $I$ or player $I I)$.

This gives a complete characterization of the pointwise relationship between determinacy and wMeas $(\mathbb{P})$. From the standard forcing notions, Sacks and Miller forcing belong to Case 1 while the other forcing notions belong to Case 2 .

Note that since $\mathrm{wMB}(\mathbb{P}) \subseteq$ wMeas $(\mathbb{P})$ for all $\mathbb{P}$, and moreover in Case 1 we have actually proved the stronger result that if $A$ is determined then it is in $\operatorname{wMB}(\mathbb{P})$, we also have a proof of the following:

- In Case 1, Determinacy implies wMB $(\mathbb{P})$ pointwise.

- In Case 2, Determinacy does not imply wMB $(\mathbb{P})$ pointwise.

The reason for the discrepancy with [Lö05] is, as we noted, due to the different definition of arboreal forcings. In [Lö05, p 1243] the author asked "it would be interesting to ask ... whether we can find a natural property of forcings (that all forcings used in applications share) that implies [that all forcing notions $\mathbb{P}$ fall under Case 1 or Case 2]". Thus, our definition of "arboreal forcings" (Definition 2.2 ) gives a solution to this question.

\section{Generalizations to $\mathbb{P}$ vs. $\mathbb{Q}$}

Although the original problem, and the conceptual question behind it, was whether determinacy has any pointwise consequences, after proving the above results it became clear that the same methods can be applied, with minimal changes, to the general situation of comparing the measurability algebras of two arboreal forcing notions $\mathbb{P}$ and $\mathbb{Q}$. The generalization of Section 4 is completely straightforward: 
Theorem 6.1. Let $\mathbb{P}$ and $\mathbb{Q}$ be arboreal. Then

1. $\operatorname{wMB}(\mathbb{P}) \nsubseteq \mathbb{M e a s}(\mathbb{Q})$,

2. $\operatorname{wMeas}(\mathbb{P}) \not \subset \operatorname{Meas}(\mathbb{Q})$,

3. $\operatorname{wMB}(\mathbb{P}) \nsubseteq \subseteq \mathrm{MB}(\mathbb{Q})$,

4. wMeas $(\mathbb{P}) \nsubseteq \operatorname{MB}(\mathbb{Q})$.

Proof. Note that by definition of arboreal forcings, it is always possible to find $P \in \mathbb{P}$ and $Q \in \mathbb{Q}$ such that $[P] \cap[Q]=\varnothing$. So fix such $P$ and $Q$ and repeat the construction in Theorem 4.3, with $[\sigma]$ replaced by $[P]$. Then the Bernstein component $A$ (and $B$ ) constructed in that proof is not in Meas $(\mathbb{Q})$ but it is disjoint from $[P]$, hence it is in $\mathrm{wMB}(\mathbb{P})$, which proves 1 . Points 2,3 and 4 follow immediately from 1 .

Note that this includes the case that $\mathbb{P}=\mathbb{Q}$, since we never needed them to be different in the argument. In particular, then, this shows that weak $\mathbb{P}$ measurability is strictly larger than $\mathbb{P}$-measurability, and similarly with the Marczewski-Burstin algebras.

Slightly less trivial is the generalization of Section 5. Here, the following notion is of central importance:

Definition 6.2. Let $\mathbb{P}$ and $\mathbb{Q}$ be arboreal. We say that $\mathbb{P}$ is thinner than $\mathbb{Q}$ if for every $Q \in \mathbb{Q}$ there exists a $P \in \mathbb{P}$ s.t. $P \subseteq Q$.

In practice, it is always easy to see whether a given $\mathbb{P}$ is thinner than $\mathbb{Q}$ : for example, Miller forcing is thinner than Laver forcing but not vice versa, Hechler forcing is thinner than Cohen forcing but not vice versa, Sacks forcing is thinner than every arboreal forcing etc.

Theorem 6.3. If $\mathbb{Q}$ is thinner than $\mathbb{P}$ then $\mathrm{wMB}(\mathbb{P}) \subseteq \mathrm{wMB}(\mathbb{Q}) \subseteq$ wMeas $(\mathbb{Q})$. Otherwise, $\mathrm{wMB}(\mathbb{P}) \nsubseteq \mathbb{w} \operatorname{Meas}(\mathbb{Q})$ and $\mathrm{wMB}(\mathbb{P}) \nsubseteq \mathbb{w M B}(\mathbb{Q})$.

Proof. If $\mathbb{Q}$ is thinner than $\mathbb{P}$, the result follows directly. If not, then by the same argument as we have used in Section 5 to prove that Case 1 and Case 2 were exhaustive, it follows that there is a $P \in \mathbb{P}$ such that $[P]$ is $\mathbb{Q}$-null. Then we repeat the construction for Case 2 from Theorem 5.5 with $[\sigma]$ replaced by $[P]$ and get a Bernstein component $A$ such that $A$ is disjoint from $[P]$ and hence in $\operatorname{wMB}(\mathbb{P})$ but $A \notin \operatorname{wMeas}(\mathbb{Q})$, and hence not in $\operatorname{wMB}(\mathbb{Q})$ either.

Of course, it would be nicer to have a full characterization, in the same vein as above, of wMeas $(\mathbb{P}) \subseteq$ wMeas $(\mathbb{Q})$. But this would involve comparing the nullideals $\mathcal{N}_{\mathbb{P}}$ with $\mathcal{N}_{\mathbb{Q}}$, and the results of [Br95] suggest that there is no general method for doing this.

The only other case that remains, is $\operatorname{Meas}(\mathbb{P}) \subseteq \operatorname{Meas}(\mathbb{Q})$. Again, $[\operatorname{Br} 95]$ suggests that there is no general method, but we can at least say the following: 
Theorem 6.4. If $\mathbb{P}$ is not thinner than $\mathbb{Q}$, then $\operatorname{Meas}(\mathbb{P}) \nsubseteq \mathbb{M e a s}(\mathbb{Q})$ (and even: $\left.\mathcal{N}_{\mathbb{P}} \nsubseteq \operatorname{Meas}(\mathbb{Q})\right)$.

Proof. If $\mathbb{P}$ is not thinner than $\mathbb{Q}$ then, by the argument that we have already seen twice, there exists some $Q \in \mathbb{Q}$ such that $[Q]$ is $\mathbb{P}$-null. Choose this $Q$, enumerate all perfect trees within $[Q]$ and, as in the proof of Theorem 4.3 find Bernstein components $A$ and $B$. Then $A \notin \operatorname{Meas}(\mathbb{Q})$ by the contraposition of Corollary 4.2 but $A \subseteq[Q] \in \mathcal{N}_{\mathbb{P}}$, so in particular $A \in \operatorname{Meas}(\mathbb{P})$.

For example, since Cohen forcing is not thinner than Hechler forcing, there is a set which is nowhere dense in the standard topology but does not have the Baire property in the dominating topology.

\section{Acknowledgements}

I would like to thank Jörg Brendle for useful discussions and Benedikt Löwe for many detailed comments, helpful ideas and critical reviews. I would also like to thank Daisuke Ikegami for pointing out Definitions 2.2 and 3.2 and, knowingly or otherwise, improving the content of this paper a great deal.

\section{References}

[BaJu95] Bartoszyński, T., Judah, H.: Set Theory, On the Structure of the Real Line. A K Peters (1995)

[Br95] Brendle, J.: Strolling Through Paradise. Fundamenta Mathematicae 148, 2643-2674 (1995)

[BrLö99] Brendle, J., Löwe, B.: Solovay-type characterizations for forcing-algebras. The Journal of Symbolic Logic 64, 1307-1323 (1999)

[El74] Ellentuck, E.: A new proof that analytic sets are Ramsey. Journal of Symbolic Logic 39, 163-165 (1974)

[Ik08] Ikegami, D.: Forcing Absoluteness and Regularity Properties. Manuscript (2008)

[Je86] Jech, T.: Multiple Forcing. Cambridge University Press, Cambridge (1986)

[Ka94] Kanamori, A.: The Higher Infinite, Large Cardinals in Set Theory from Their Beginnings. Springer-Verlag, Berlin (2004)

[Lö05] Löwe, B.: The pointwise view of determinacy: arboreal forcings, measurability, and weak measurability. Rocky Mountain Journal of Mathematics 35, 1233-1249 (2005)

[So70] Solovay, R. M.: A model of set theory in which every set of reals is Lebesgue measurable. Annals of Mathematics Ser 2. 92, 1-56 (1970) 